\title{
Comparison of Available Methods for Investigating the in vitro Activity of Colistin Against Different Gram-Negative Bacilli
}

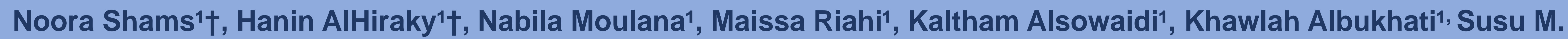 Zughaier ${ }^{2}$, Nahla O. Eltai ${ }^{3 *}$}

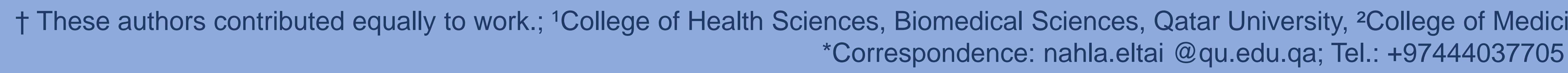

\section{ABSTRACT}

The surge in the prevalence of multidrug-resistant (MDR) Gram-negative bacterial infections led to the reuse of colistin as a treatment for MDR pathogens. This study aimed to determine economical, simple, and reliable colistin susceptibility testing methods as an alternative to the time and effort-consuming microdilution technique. Seven colistin susceptibility-testing methods were examined on 63 isolates, and the data were analyzed using different statistical methods. Results showed that disk diffusion is still an ineffective method for measuring colistin susceptibility to Gram-negative Bacilli. E-test, CompASPTM SensiTest, and Phoenix ID/AST automated colistin methods are similar to the current recommended broth microdilution procedure in terms of reproducibility, sturdiness, and simplicity. Our study revealed that E-test and ComASPTM SensiTest and Phoenix techniques are recommended for routine laboratories with a large workload.

\section{INTRODUCTION}

Colistin is the last resort for multidrug resistance Gram-negative Bacilli (MDR-GNB), mainly against Enterobacteriaceae $(1,2)$. Several MDR-GNB bacteria developed their defence mechanisms against colistin. Broth microdilution is recommended method by both the Clinical and Laboratory Standards Institute (CLSI) and the European Committee on Antimicrobial Susceptibility Testing (EUCAST) for Colistin susceptibility testing (3). However, this method is impractical for clinical laboratories due to the large sample load in routine microbiology laboratories (4). Thus, an alternative, more practical method is needed. The study outcomes will provide a framework for clinical laboratories to select the appropriate method to test for colistin resistance by comparing the different colistin susceptibility testing methods on some gram-negative bacilli to find the feasible colistin test.

\section{OBJECTIVES}

To compare seven methods of colistin susceptibility testing to the gold standard broth microdilution. These methods include Disk diffusion, E-test,

CHROMagarTM COL-APSE, ComASPTM SensiTest Colistin, broth disk elution, Colistin agar test, and BD Phoenix ID/AST.

To identify the colistin resistance's genetic determinants to find if it affects the phenotypic testing method.

\section{METHODOLOGY}

(1) Bacteria sample
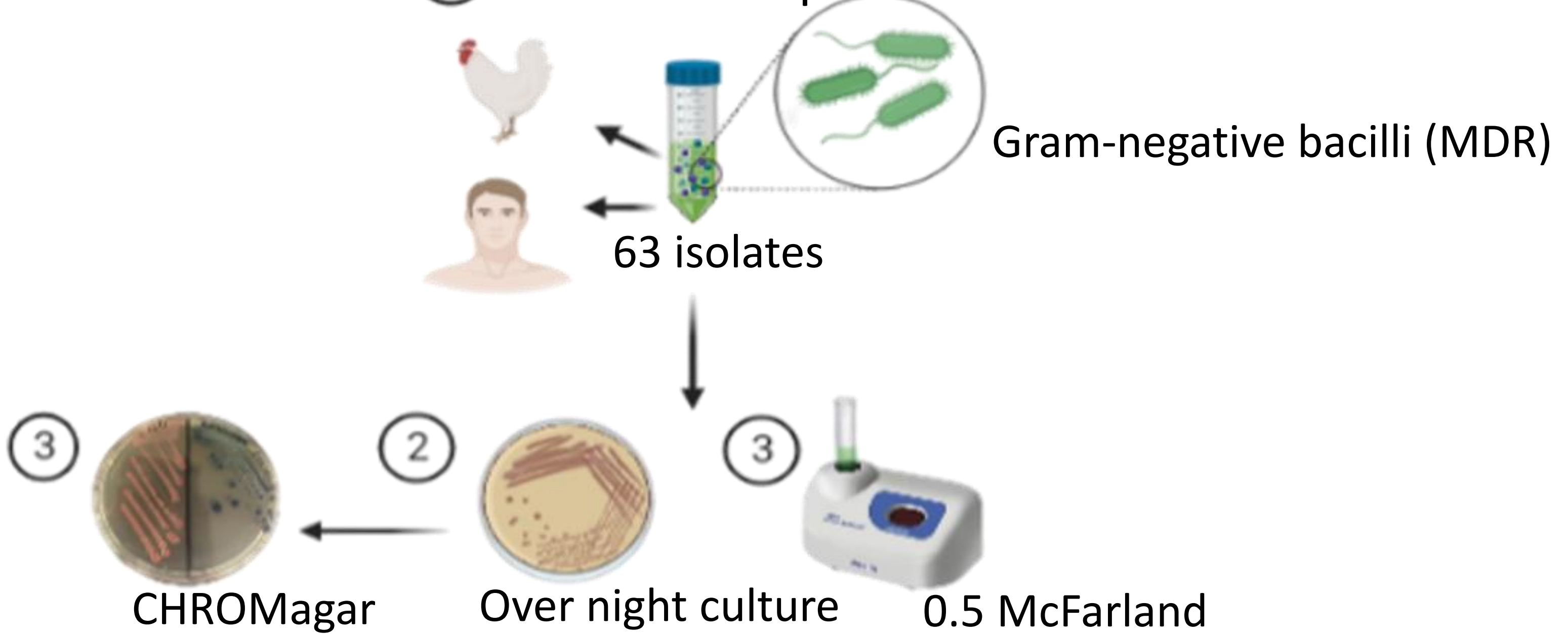

(4)

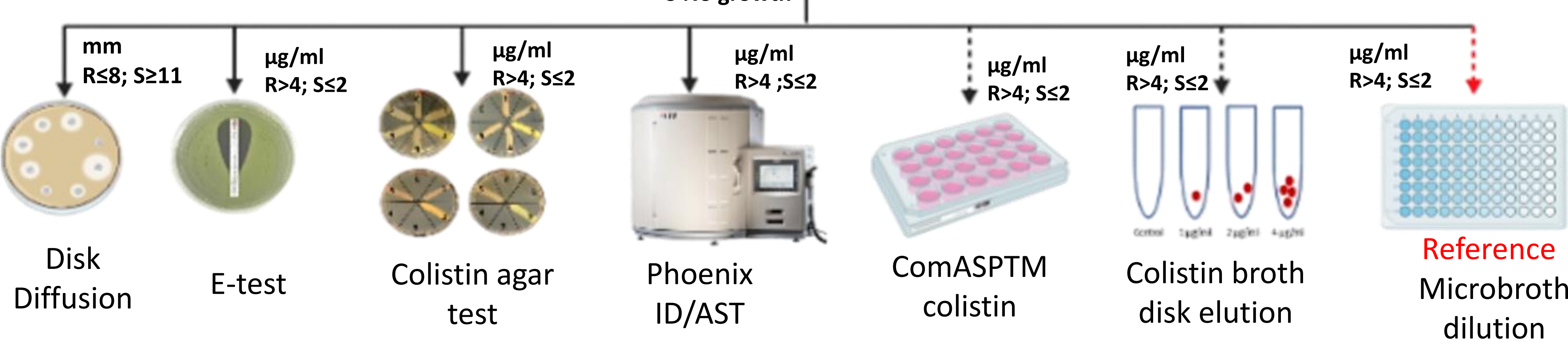

Figure 1. Comprehensive summery of methods done for comparison the MIC of colistin with reference method made by BioRender.com.

\section{Whole genome sequencing}

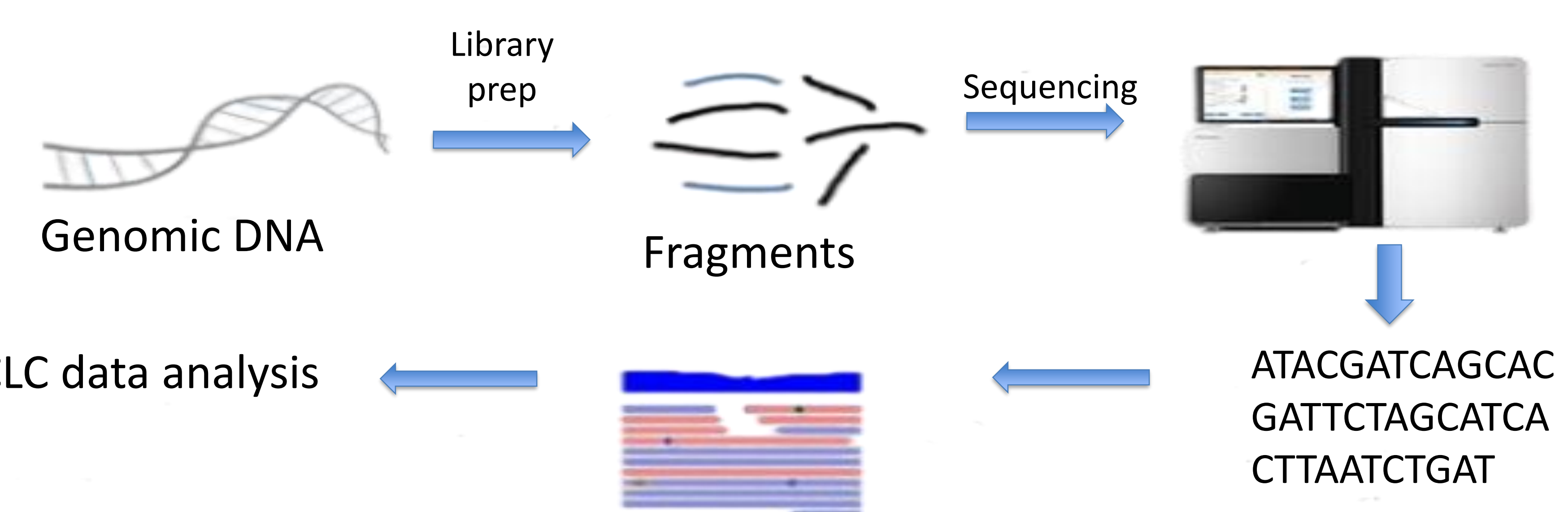

Align reads to reference

Figure 2. Whole genome sequencing procedure by illumine next generation sequence made by BioRender.com

\section{RESULTS}

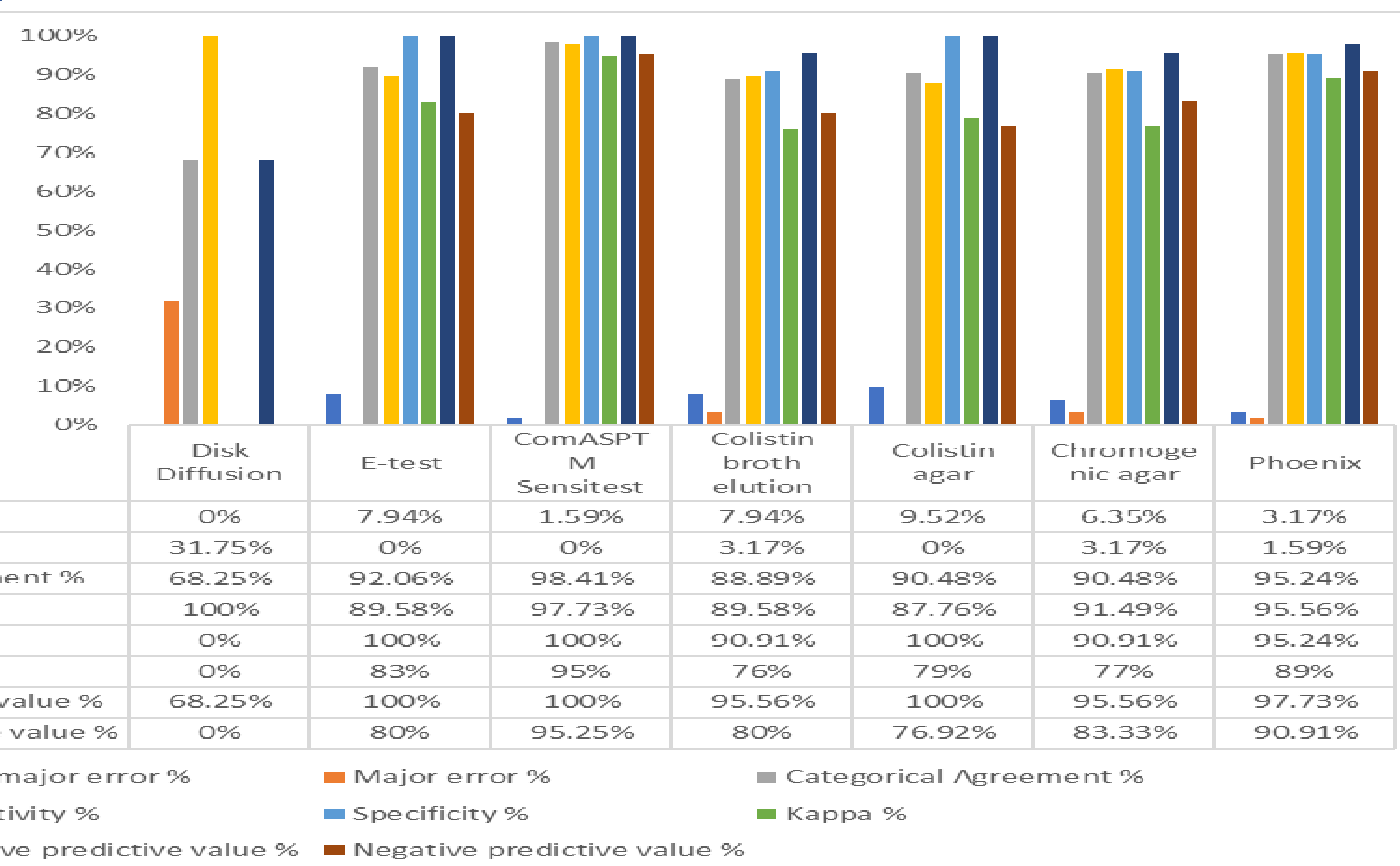

Figure 3. Comparative evaluation of seven colistin diagnostic tests by categorical agreement, Kappa, sensitivity, and specificity values compared to the gold- standard method (BMD).

\section{CONCLUSIONS}

The results of this study suggest that Phoenix, E-test and CompASPT SensiTest colistin methods have remained superior in terms of reproducibility, sturdiness, and simplicity of use with a performance similar to the current recommended BMD procedure.

These methods can be used as an alternative to the current laborious, impractical broth microdilution technique

This study did not ensure any relation between the type of colistin resistance genetic determinant (chromosomal/plasmid-mediated) and the performance of the specific colistin susceptibility test.

Disk diffusion is still an ineffective method for measuring colistin susceptibility to Gram-negative Bacilli.

The disk diffusion method showed low values for all the statistical analyses. In contrast, the highest values of sensitivity, specificity, categorical agreement, kappa value, positive predictive value, and negative predictive value were reported on Phoenix, ComASPTM sensitest, and E-test methods compared with the microbroth dilution reference method. Our study did not ensure any relation between the type of colistin resistance genetic determinant (chromosomal/plasmid-mediated) and the performance of the specific colistin susceptibility test.

\begin{tabular}{l|ccccc}
\hline Organism & $\begin{array}{l}\text { Number of } \\
\text { isolates }\end{array}$ & \multicolumn{4}{c}{ Colistin resistance type } \\
\cline { 3 - 6 } & & $m c r l-1$ & GMR & $m c r 1-1 \&$ GMR & Not preser \\
\cline { 3 - 7 } Escherichia coli & 43 & 26 & 2 & 1 & 14 \\
Klebsiella pneumoniae & 18 & 0 & 11 & 2 & 5 \\
Pseudomonas aeruginosa & 2 & 0 & 2 & 0 & 0 \\
Total & 63 & 26 & 15 & 3 & 19 \\
\hline
\end{tabular}

Figure 4. A table shows the genetic determinant of colistin resistance among 63 Gram-negative Bacilli isolates.

\section{ACKNOWLEDGEMENTS}

This study was supported by (UREP26024-1-002) grant from the Qatar National Research Fund (QNRF)

Authors would like to thank Hana Abdelrahman and Lubna Abu rub for the technical assistance. Also, we thank Dr. Andres Perez Lopez, who kindly allow us to test colistin resistance using $\mathrm{BD}$ Phoenix ID/AST at Sidra Medicine.

\section{REFERENCES}

Carretto E, Brovarone F, Russello G, Nardini P, El-Bouseary MM Aboklaish AF, et al. Clinical Validation of SensiTest Colistin, a Broth To Evaluate Colistin MICs. Journal of Clinical Microbiology 2018;56(4).

Gwozdzinski K, Azarderakhsh S, Imizalioglu C, Falgenhauer L, Chozdinski K, Azarderakhs S, Inirzalioglu C, Falgenhauer $L$ esting. Journal of Clinical Microbiology. 2018;56(5). WHO, The detection and reporting of colistin resistance. 2018 License: CC BY-NC-SA 3.0 IGO.

Sharma: A A Resistant Gram -Negative Bacilli; Comparative Study of E-test and Vitek 2 Compact $^{\text {TM }}$ with Broth Microdilution. Galore International Journal of Health Sciences and Research. 2019;4(4):110-5. 